\title{
Erratum to: Subnormality of Generalized Creation Operators on Bargmann's Space of an Infinite Order
}

\author{
Jerzy Bartłomiej Stochel
}

\begin{abstract}
The purpose of this note is to correct an error in [1].
Mathematics Subject Classification (2010). Primary 47B20; Secondary 47B32.
\end{abstract}

Keywords. Creation operator, Bargmann Hilbert space, subnormality.

This is a correction in the proof to Lemma 6 of [1]. We begin by replacing the incorrect formula in the fifth line of this proof by the following one

$$
\left(\left.A_{a}^{+}\right|_{\mathcal{M}}\right) f^{n}=\left(A_{a}^{1} f\right)(n)+\left(A_{a}^{2} f\right)(n), \quad n \in \mathbb{N},
$$

where

$$
\begin{aligned}
\left(A_{a}^{1} f\right)(n) & :=\sum_{\beta \in \pi_{1}(n)}\left[\sum_{i=1}^{n} f_{\beta-\delta_{i}} \bar{a}_{i} \sqrt{\beta_{i}}\right] e_{\beta}, \\
\left(A_{a}^{2} f\right)(n) & :=\sum_{\beta \in \pi_{2}(n)}\left[\sum_{i=n+1}^{\infty} f_{\beta-\delta_{i}} \bar{a}_{i} \sqrt{\beta_{i}}\right] e_{\beta}, \\
\pi_{1}(n) & :=\Im(n, 0, n+1) \text { and } \pi_{2}(n):=\Im(n, 1, n+1) .
\end{aligned}
$$

Clearly $\pi_{1}(n) \cap \pi_{2}(n)=\emptyset$ and $\pi_{1}(n) \subset \pi_{1}(n+1)$ for all $n \in \mathbb{N}$, and $\bigcup_{n \in \mathbb{N}} \pi_{1}(n)=\Im$. Since $f \in D\left(A_{a}^{+}\right)$, the sum $\sum_{\beta \in \Im}\left|\sum_{i=1}^{\infty} f_{\beta-\delta_{i}} \bar{a}_{i} \sqrt{\beta_{i}}\right|^{2}$ is finite. Hence

$$
\begin{aligned}
\left(A_{a}^{1} f\right)(n) & :=\sum_{\beta \in \pi_{1}(n)}\left[\sum_{i=1}^{\infty} f_{\beta-\delta_{i}} \bar{a}_{i} \sqrt{\beta_{i}}\right] e_{\beta} \stackrel{n \rightarrow \infty}{\longrightarrow} \sum_{\beta \in \Im}\left[\sum_{i=1}^{\infty} f_{\beta-\delta_{i}} \bar{a}_{i} \sqrt{\beta_{i}}\right] e_{\beta} \\
& =A_{a}^{+} f .
\end{aligned}
$$




\section{Moreover}

$$
\begin{aligned}
\left\|A_{a}^{2}(n)\right\|^{2} & :=\sum_{\beta \in \pi_{2}(n)}\left|\sum_{i=n+1}^{\infty} f_{\beta-\delta_{i}} \bar{a}_{i} \sqrt{\beta_{i}}\right|^{2} \\
& =\sum_{\beta \in \pi_{2}(n)}\left|f_{p_{n}(\beta)} \bar{a}_{i(\beta)}\right|^{2}=\sum_{\substack{\alpha \in Z_{+}^{n} \\
|\alpha| \leq n}} \sum_{i=n+1}^{\infty}\left|f_{\alpha}\right|^{2}\left|a_{i}\right|^{2} \\
& \left.=\left(\sum_{\substack{\alpha \in Z_{+}^{n} \\
|\alpha| \leq n}}\left|f_{\alpha}\right|^{2}\right)\left(\sum_{i=n+1}^{\infty}\left|a_{i}\right|^{2}\right) \stackrel{n \rightarrow \infty}{\longrightarrow} 0 \text { (because } f \in B \text { and } a \in l^{2}\right),
\end{aligned}
$$

where $p_{n}(\beta):=\left(\beta_{1}, \ldots, \beta_{n}, 0,0, \ldots\right)$ and $i(\beta) \in \mathbb{N}$ is such that

$$
\beta=\left(\beta_{1}, \ldots, \beta_{n}, 0, \ldots, 0, \underset{i(\beta)}{1}, 0,0, \ldots\right) .
$$

As a consequence, we have

$$
A_{a}^{2}(n) \rightarrow 0 \text { as } n \rightarrow \infty .
$$

It follows from (5.12.1), (5.12.2) and (5.12.3) that $\left(\left.A_{a}^{+}\right|_{\mathcal{M}}\right) f^{n} \rightarrow A_{a}^{+} f$ in $B$, and thus the pair $\left(f, A_{a}^{+} f\right)$ belongs to the graph of $\overline{\left.A_{a}^{+}\right|_{\mathcal{M}}}$. This completes the proof.

Open Access. This article is distributed under the terms of the Creative Commons Attribution License which permits any use, distribution, and reproduction in any medium, provided the original author(s) and the source are credited.

\section{Reference}

[1] Stochel, J.B.: Subnormality of generalized creation operators on Bargmann's space of an infinite order. Integr. Equat. Oper. Th. 15, 1011-1032 (1992)

Jerzy Bartłomiej Stochel $(\bowtie)$

Department of Applied Mathematics

AGH University of Science and Technology

Al. Mickiewicza 30

30-059 Cracow

Poland

e-mail: stochel@agh.edu.pl 